\title{
AbDelouahab Arouche
}

\section{Sur la complétion de la $K$-théorie équivariante}

Annales de la faculté des sciences de Toulouse $6^{e}$ série, tome $6, \mathrm{n}^{\circ} 3$ (1997), p. 377-387

<http://www.numdam.org/item?id=AFST_1997_6_6_3_377_0>

(C) Université Paul Sabatier, 1997, tous droits réservés.

L'accès aux archives de la revue «Annales de la faculté des sciences de Toulouse » (http://picard.ups-tlse.fr/ annales/) implique l'accord avec les conditions générales d'utilisation (http://www.numdam.org/conditions). Toute utilisation commerciale ou impression systématique est constitutive d'une infraction pénale. Toute copie ou impression de ce fichier doit contenir la présente mention de copyright.

\section{NumDam}

Article numérisé dans le cadre du programme Numérisation de documents anciens mathématiques http://www.numdam.org/ 


\title{
Sur la complétion de la $K$-théorie équivariante ${ }^{(*)}$
}

\author{
Abdelouahab Arouche ${ }^{(1)}$
}

RÉSUMÉ. - Pour un foncteur $M$ défini sur la catégorie des orbites d'un groupe de Lie compact $\Gamma$ et une famille $\mathcal{F}$ de sous-groupes de $\Gamma$, on calcule dans [J] la $I^{M}(\mathcal{F})$-complétion de la $K$-théorie équivariante d'un $\Gamma$-espace $X$ comme étant égale à $K_{\Gamma}^{*}(X \times E \mathcal{F})$ où $E \mathcal{F}$ est un objet final de la catégorie homotopique des $\Gamma$-espaces $\mathcal{F}$-énumérables. D'abord, on étudie la relation entre de telles complétions pour des foncteurs $M$ différents. Ensuite, un exemple d'un tel foncteur est donné en se basant sur le travail de $[B]$.

\begin{abstract}
It has been shown in [J], that for a functor $M$ defined on the category of orbits of a Lie group $\Gamma$ and a family $\mathcal{F}$ of subgroups of $\Gamma$, the equivariant $K$-theory of a $\Gamma$-space $X$, completed with respect to the $I^{M}(\mathcal{F})$-topology, is equal to $K_{\Gamma}^{*}(X \times E \mathcal{F})$ where $E \mathcal{F}$ is a terminal object in the category of $\mathcal{F}$-numerable $\Gamma$-spaces. First, we study the relation between such completions for different functors $M$. Then, we give an example of such a functor based on the work of [B].
\end{abstract}

\section{Famille de sous-groupes et complétion}

Soit $\Gamma$ un groupe de Lie compact. Un ensemble $\mathcal{F}$ de sous-groupes (fermés) est dit famille s'il est stable par sous-conjugaison.

Un $\Gamma$-espace est dit $\mathcal{F}$-énumérable s'il admet un $\Gamma$-recouvrement ouvert énumérable $\mathcal{U}$ tel que pour tout $U \in \mathcal{U}$, il existe une application équivariante $U \rightarrow \Gamma / \Lambda$, pour un $\Lambda \in \mathcal{F}$. La catégorie homotopique des $\Gamma$-espaces $\mathcal{F}$ énumérables admet un objet final noté $E \mathcal{F}$.

(*) Reçu le 5 mai 1995

(1) King Saud University, College of Sciences, Department of Mathematics, P. O. Box : 2455, Riyadh 11451 (Saudi Arabia)

e-mail : F40M014@KSU.EDU.SA 
Étant donnée une théorie de cohomologie équivariante $h_{\Gamma}$, définie sur la catégorie des $\Gamma$-espaces compacts, on définit une théorie de procohomologie $h_{\Gamma}[\mathcal{F}]$ sur la même catégorie et ce, pour toute famille $\mathcal{F}$ de sous-groupes de $\Gamma:$

$$
h_{\Gamma}[\mathcal{F}](X)=\left\{h_{\Gamma}(X \times K) \mid K \subset E \mathcal{F}, K \text { compact }\right\} .
$$

Soit $M: \mathcal{O}_{\Gamma} \rightarrow \mathcal{A}^{*}$ un foncteur contravariant défini sur la catégorie des orbites de $\Gamma$ et à valeurs dans la catégorie des anneaux gradués. On notera $M_{\Lambda}$ sa valeur sur $\Gamma / \Lambda$. On définit l'idéal :

$$
I_{\Lambda}^{M}=\operatorname{ker}\left\{M_{\Gamma} \rightarrow M_{\Lambda}\right\} .
$$

Alors la $\mathcal{F}$-topologie sur tout $M_{\Gamma^{-}}$-module n'est autre que la topologie $I^{M}(\mathcal{F})$-adique, où

$$
I^{M}(\mathcal{F})=\left\{I_{\Lambda}^{M} \mid \Lambda \in \mathcal{F}\right\}
$$

On montre (cf. [J, $\S 1.8]$ ) que pour un $\Gamma$-espace compact quelconque, la projection $X \times E \mathcal{F} \rightarrow X$ définit un homomorphisme de pro-anneaux

$$
p_{X}(\mathcal{F}): h_{\Gamma} / I^{M}(\mathcal{F})(X) \longrightarrow h_{\Gamma}[\mathcal{F}](X) .
$$

Dans la suite, on prendra pour théorie de cohomologie la $K$-théorie équivariante $K_{\Gamma}^{*}$. Rappelons que pour tout $\Gamma$-espace (compact) $X, K_{\Gamma}^{*}(X)=$ $K_{\Gamma}^{0}(X) \oplus K_{\Gamma}^{1}(X)$ où $K_{\Gamma}^{0}(X)$ est le groupe de Grothendieck des $\Gamma$-fibrés vectoriels complexes au-dessus de $X$ et où $K_{\Gamma}^{1}(X)$ est défini à partir de $\Gamma$ fibrés au-dessus de la suspension de $X . K_{\Gamma}^{*}(X)$ est un anneau $\mathbb{Z}_{2}$-gradué (cf. [S1]).

Soit $Z$ un $\Gamma$-espace compact fixé et $\Lambda \subset \Gamma$ un sous-groupe. Considérons la catégorie $\mathcal{C}_{Z}^{\Lambda}$ dont les objets sont $\Lambda$-espaces $X$ munis d'une $\Lambda$-application $X \stackrel{\sigma}{\rightarrow} Z$. Un morphisme entre deux objets $(X, \sigma)$ et $(Y, \tau)$ est une $\Lambda$ application $X \stackrel{f}{\rightarrow} Y$ telle que $\sigma=\tau \circ f$. Par exemple, si $(X, \sigma)$ est un objet de $\mathcal{C}_{Z}^{\Lambda}$, alors pour tout $\Lambda$-espace $Y,\left(X \times Y, \sigma \circ \mathrm{pr}_{1}\right)$ est aussi un objet $\operatorname{de} \mathcal{C}_{Z}^{\Lambda}$ et $\operatorname{pr}_{1}: X \times Y \rightarrow X$ est un morphisme dans $\mathcal{C}_{Z}^{\Lambda}$. Un objet de $\mathcal{C}_{Z}^{\Gamma}$ peut être considéré comme objet de $\mathcal{C}_{Z}^{\Lambda}$ pour tout sous-groupe $\Lambda \subset \Gamma$.

Si l'on pose

$$
M_{\Lambda}=K_{\Lambda}^{*}(Z),
$$

alors $K_{\Gamma}^{*}$ est un module sur $M(=M(Z))$, c'est-à-dire pour tout sous-groupe $\Lambda \subset \Gamma$ et tout objet $X$ de $\mathcal{C}_{Z}^{\Lambda}, K_{\Lambda}^{*}(X)$ est un $M_{\Lambda}$-module de telle sorte que la condition de compatibilité suivante soit vérifiée. 
Pour tout objet $X \operatorname{de} \mathcal{C}_{Z}^{\Gamma}$ et tout sous-groupe $\Lambda$ de $\Gamma$, le carré suivant est commutatif :

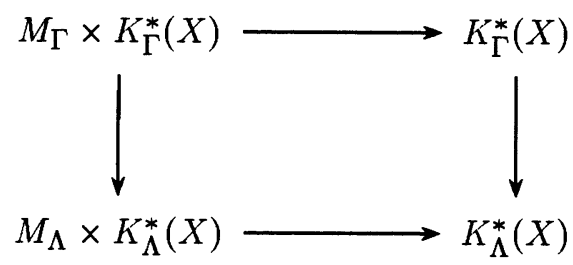

Supposons $Z$ tel que les deux conditions suivantes soient vérifiées, pour toute famille $\mathcal{F}$ de sous-groupes de $\Gamma$ :

$(N)$ l'anneau $M_{\Gamma}$ est noethérien et pour tout sous-groupe $\Lambda \subset \Gamma$, l'homomorphisme $M_{\Gamma} \rightarrow M_{\Lambda}$ fait de $M_{\Lambda}$ un $M_{\Gamma \text {-module de type }}$ fini;

$\left(R_{\mathcal{F}}\right)$ pour tout sous-groupe $\Lambda \subset \Gamma$, la $\mathcal{F}$-topologie définie sur $M_{\Lambda}$ par la restriction $M_{\Gamma} \rightarrow M_{\Lambda}$ coïncide avec la $\mathcal{F} \cap \Lambda$-topologie sur $M_{\Lambda}$; c'est-à-dire celle définie par les idéaux

$$
\left\{\operatorname{ker}\left(M_{\Lambda} \rightarrow M_{\Delta}\right) \mid \Delta \in \mathcal{F} \cap \Lambda\right\} .
$$

On a alors le résultat suivant.

THÉORÈme 1.1.- Soit $X$ un objet de $\mathcal{C}_{Z}^{\Gamma}(X)$ tel que $K_{\Lambda}^{*}(X)$ soit un $M_{\Lambda}$-module de type fini, pour tout sous-groupe $\Lambda \subset \Gamma$. Alors, pour toute famille $\mathcal{F}$ de sous-groupes de $\Gamma$, l'application

$$
p_{X}(\mathcal{F}):\left(K_{\Gamma}^{*} / I^{M}(\mathcal{F})\right)(X) \longrightarrow K_{\Gamma}^{*}[\mathcal{F}](X)
$$

est un isomorphisme de pro-anneaux.

Preuve. - D'après [J, $\S 2.2]$, il suffit de montrer que, pour tout sousgroupe $\Lambda \subset \Gamma$, le morphisme $p_{X}\left(\mathcal{P}_{\Lambda}\right)$ est un isomorphisme, où $\mathcal{P}_{\Lambda}$ est la famille des sous-groupes propres de $\Lambda$. $\grave{A}$ cette fin, pour un sous-groupe $\Lambda \subset \Gamma$, considérons l'ensemble $\mathcal{V}$ de toutes les représentations de $\Lambda$ sans facteur trivial. Alors $\mathcal{V}$ définit la famille de sous-groupes de $\Lambda$ :

$$
\mathcal{F}_{\mathcal{V}}=\bigcup_{V \in \mathcal{V}}\left\{\Delta \leq \Lambda \mid V^{\Delta} \neq\{0\}\right\}
$$

La preuve du théorème 1.1 est contenue dans les paragraphes 1.2 à 1.5. 


\section{Abdelouahab Arouche}

LEMME 1.2. $-\mathcal{P}_{\Lambda}=\mathcal{F}_{\mathcal{V}}$

Preuve. - Soit $\Delta \in \mathcal{P}_{\Lambda}$. Il existe une représentation $V$ de $\Lambda$ et un point $v$ de $V$ tels que $\Delta=\Lambda_{v}$ (cf. [D, $\S$ I.5.5]). Ceci entraîne que $v \neq 0$, car $\Lambda_{0}=\Lambda$ et $\Delta$ est propre. D'où, $V^{\Delta} \neq 0$. On peut supposer $V$ sans facteur trivial, quitte à l'en débarrasser. D'où, $\Delta \in \mathcal{F}_{\mathcal{V}}$. Réciproquement, soit $\Delta \in \mathcal{F}_{\mathcal{V}}$. Il existe donc $V \in \mathcal{V}$ tel que $V^{\Delta} \neq 0$. Si $\Delta=\Lambda, V$ aurait donc un facteur trivial, d'où la contradiction. Donc, $\Delta \in \mathcal{P}_{\Lambda}$.

Proposition 1.3. - La topologie définie par la famille d'idéaux principaux $\left\{e(V) \cdot K_{\Lambda}^{*}(Z) \mid V \in \mathcal{V}\right\}$ sur $K_{\Lambda}^{*}(Z)$, ò̀ $e(V)$ est la classe d'Euler de $V$, coïcide avec la $\mathcal{F}_{\mathcal{V}}$-topologie.

Preuve. - Voir [A] où l'on a une généralisation de [J, § 3.1].

LEMME 1.4. - Si $\mathcal{V}=\left\{V_{i}\right\}_{i \in \mathbb{N}^{*}}$, alors

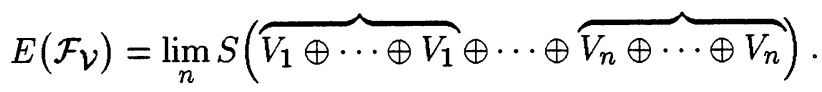

Preuve. - Voir [J].

THÉORÈME 1.5. - Dans les conditions du théorème 1.1,

$$
p_{X}\left(\mathcal{F}_{\mathcal{V}}\right):\left(K_{\Lambda}^{*} / I^{M}\left(\mathcal{F}_{\mathcal{V}}\right)\right)(X) \longrightarrow K_{\Lambda}^{*}\left[\mathcal{F}_{\mathcal{V}}\right](X)
$$

est un isomorphisme de pro-anneaux.

Preuve. - (d'après $[\mathrm{J}])$ D'après le lemme précédent,

$$
K_{\Lambda}^{*}\left[\mathcal{F}_{\mathcal{V}}\right](X)=\left\{K_{\Lambda}^{*}\left(X \times S V^{n}\right) \mid n \in \mathbb{N}^{*}\right\}
$$

où

$$
V^{n}:=\overbrace{V_{1} \oplus \cdots \oplus V_{1}}^{\cdots} \oplus \oplus \overbrace{V_{n} \oplus \cdots \oplus V_{n}} .
$$

La suite exacte de Gysin,

$$
\ldots \stackrel{\delta}{\longrightarrow} K_{\Lambda}^{*}(X) \stackrel{e\left(V^{n}\right)}{\longrightarrow} K_{\Lambda}^{*}(X) \stackrel{\pi^{*}}{\longrightarrow} K_{\Lambda}^{*}\left(S V^{n} \times X\right) \stackrel{\delta}{\longrightarrow} \ldots
$$

donne lieu à la suite exacte courte :

$$
0 \longrightarrow K / e\left(V^{n}\right) \cdot K \longrightarrow K_{\Lambda}^{*}\left(S V^{n} \times X\right) \longrightarrow{ }_{e\left(V^{n}\right)} K \longrightarrow 0
$$

où $K:=K_{\Lambda}^{*}(X)$ et $e\left(V^{n}\right) K:=\left\{x \in K: e\left(V^{n}\right) x=0\right\}$. 
Pour prouver le théorème, on doit trouver, pour chaque $n$, un entier $k$ et un morphisme $\beta_{n}: K_{\Lambda}^{*}\left(S V^{n+k} \times X\right) \rightarrow K / e\left(V^{n}\right) \cdot K$ tels que le diagramme suivant commute :

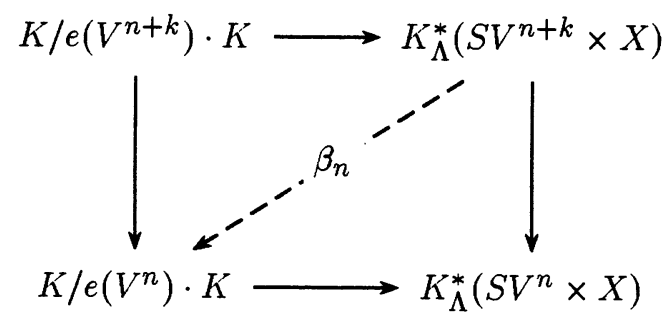

Or on a le diagramme commutatif suivant, dont les lignes sont exactes :

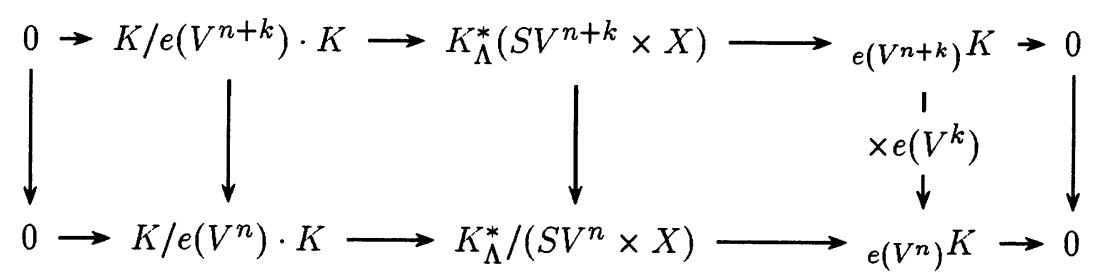

$K$ étant de type fini sur $K_{\Lambda}^{*}(Z)$, on peut trouver un rang $k$ tel que $e\left(V^{k}\right) K=e\left(V^{n+k}\right) K$ pour tout $n$. Donc $e\left(V^{k}\right)$ annule $e\left(V^{n+k}\right) K$, d'où l'existence de $\beta_{n}$.

Corollaire 1.6. - Si, en plus des conditions $(N)$ et $\left(R_{\mathcal{F}}\right), Z$ est tel que $M_{\Lambda}$ soit noethérien (comme anneau), pour tout sous-groupe $\Lambda \subset \Gamma$, alors $p_{Z}(\mathcal{F})$ est un isomorphisme pour toute famille $\mathcal{F}$ de sous-groupes de $\Gamma$. C'est le cas, par exemple, si $Z$ a un point fixe et vérifie : $M_{\Lambda}$ est un $R(\Lambda)$-module de type fini, pour tout sous-groupe $\Lambda \subset \Gamma$.

Corollaire 1.7.- Soit $Z$ un $\Gamma$-espace compact tel que $M$ vérifie les conditions $(N)$ et $\left(R_{\mathcal{F}}\right)$. Supposons que $Z$ admet un point fixe noté + . Alors $p_{+}(\mathcal{F})$ est un isomorphisme pour toute famille $\mathcal{F}$ de sous-groupes de $\Gamma$.

Considérons le foncteur $L:=M$ (point). Dans [J], on montre que $L$ vérifie les conditions $(N)$ et $\left(R_{\mathcal{F}}\right)$ pour toute $\mathcal{F}$. On a alors (cf. [J]) le théorème 1.1 où l'on remplace $M_{\Lambda}$ par $R(\Lambda)$, anneau des représentations complexes de $\Lambda$. D'où le résultat qui suit. 
Proposition 1.8. - Soit $Z$ un $\Gamma$-espace compact tel que $M=M(Z)$ vérifie les conditions $(N)$ et $\left(R_{\mathcal{F}}\right)$ pour toute famille $\mathcal{F}$ de sous-groupes de $\Gamma$. Si un objet $X$ de $\mathcal{C}_{Z}^{\Gamma}$ est tel que $K_{\Lambda}^{*}(X)$ est de type fini à la fois sur $M_{\Lambda}$ et $L_{\Lambda}$, pour tout sous-groupe $\Lambda \subset \Gamma$, alors, pour toute famille $\mathcal{F}$, on a un isomorphisme de pro-systèmes :

$$
\left(K_{\Gamma}^{*} / I^{M}(\mathcal{F})\right)(X) \cong\left(K_{\Gamma}^{*} / I^{L}(\mathcal{F})\right)(X) .
$$

Si $Z$ est muni d'un point de base et si $M_{\Lambda}$ est de type fini sur $L_{\Lambda}, \forall \Lambda \subset \Gamma$, il suffit alors que $K_{\Lambda}^{*}(X)$ soit de type fini sur $L_{\Lambda}, \forall \Lambda \subset \Gamma$. Dans ce dernier cas, on a, en particulier :

$$
\left(K_{\Gamma}^{*} / I^{M}(\mathcal{F})\right)(Z) \cong\left(K_{\Gamma}^{*} / I^{L}(\mathcal{F})\right)(Z)
$$

et

$$
R(\Gamma) / I^{M}(\mathcal{F}) \cong R(\Gamma) / I^{L}(\mathcal{F})
$$

Remarque. - Examinons de près le dernier cas : si $Z$ est muni d'un point de base, alors $L_{\Lambda}$ est un sous-anneau de $M_{\Lambda}, \forall \Lambda \subset \Gamma$. On en déduit que

$$
I_{\Lambda}^{L} \subset I_{\Lambda}^{M}, \quad \forall \Lambda \subset \Gamma .
$$

En d'autres termes, la topologie $I^{L}(\mathcal{F})$-adique sur tout $M_{\Gamma}$-module est plus fine que la $I^{M}(\mathcal{F})$-adique . La proposition 1.8 affirme qu'elles sont en fait identiques, car les deux sont aussi définies par la famille d'idéaux (cf. [AS, $\S 2.3])$

$$
\left\{J_{K}=\operatorname{ker}\left(K_{\Gamma}^{*}(X) \longrightarrow K_{\Gamma}^{*}(X \times K)\right)\right\} .
$$

Corollaire 1.9. - Soit $Z$ un $\Gamma$-espace compact muni d'un point de base tel que $K_{\Lambda}^{*}(Z)$ soit un $R(\Lambda)$-module de type fini pour tout sous-groupe $\Lambda \subset \Gamma$. Supposons que le foncteur $M=M(Z)$ vérifie la condition $\left(R_{\mathcal{F}}\right)$, $\forall \mathcal{F}$. Alors, si $X$ est un $\Gamma$-espace compact tel que $K_{\Lambda}^{*}(X)$ soit de type fini sur $R(\Lambda)$, pour tout sous-groupe $\Lambda \subset \Gamma$, on a un isomorphisme :

$$
K_{\Gamma}^{*}(X)_{\hat{I}^{M}(\mathcal{F})} \cong K_{\Gamma}^{*}(X)_{\hat{I}^{L}(\mathcal{F})},
$$

pour toute famille $\mathcal{F}$ de sous-groupes de $\Gamma$. En particulier, on a les deux isomorphismes :

$$
K_{\Gamma}^{*}(Z)_{\hat{I}^{M}(\mathcal{F})} \cong K_{\Gamma}^{*}(Z)_{\hat{I}^{L}(\mathcal{F})} \text { et } R(\Gamma)_{\hat{I}^{M}(\mathcal{F})} \cong R(\Gamma)_{\hat{I}^{L}(\mathcal{F})}
$$


où $\hat{I}^{M}(\mathcal{F})$ (resp. $\hat{I}^{L}(\mathcal{F})$ ) désigne la complétion par rapport à la topologie $I^{M}(\mathcal{F})$-adique (resp. $I^{L}(\mathcal{F})$-adique).

Preuve. - La condition $(N)$ est automatique (cf. [AM, $\S 7.2]$ ), et un isomorphisme entre pro-systèmes entraîne un isomorphisme entre limites projectives.

\section{2. Étude des conditions $(N)$ et $\left(R_{\mathcal{F}}\right)$}

Soit $Z$ un $\Gamma$ espace compact muni d'un point de base.

La condition $(N)$ est satisfaite si $Z$ est une variété différentiable sur laquelle $\Gamma$ agit différentiablement. Car alors, $K_{\Lambda}^{*}(Z)$ est de type fini sur le sous-anneau noethérien $R(\Lambda)$ pour tout sous-groupe $\Lambda \subset \Gamma[\mathrm{S} 1, \S 5.4]$.

Un autre cas où la condition $(N)$ est satisfaite est celui d'un espace compact $Z$ sur lequel $\Gamma$ opère trivialement et tel que $K^{*}(Z)$ soit noethérien. En effet, dans ce cas $K_{\Lambda}^{*}(Z) \cong K^{*}(Z) \otimes R(\Lambda)$ pour tout sous-groupe $\Lambda \subset \Gamma$ [S1, $\S 2.2]$. Comme $R(\Gamma)$ est de type fini comme $\mathbb{Z}$-algèbre [S2, $\S 3.3], K_{\Gamma}^{*}(Z)$ est de type fini comme $K^{*}(Z)$-algèbre et est donc noethérien [AM, $\left.\S 7.7\right]$. De plus, $R(\Lambda)$ est un $R(\Gamma)$-module de type fini pour tout $\Lambda \leq \Gamma[S 2, \S 3.2]$, donc la restriction $M_{\Gamma} \rightarrow M_{\Lambda}$ fait de ce dernier un $M_{\Gamma}$-module de type fini.

Hodgkin a construit dans $[\mathrm{H}]$ une suite spectrale

$$
E_{2}^{* *}=\operatorname{Tor}_{R(\Gamma)}^{* *}\left(K_{\Gamma}^{*}(X), K_{\Gamma}^{*}(Y)\right) \Longrightarrow F_{\Gamma}^{*}(X, Y)
$$

et a montré que dans certains cas, par exemple quand $\pi_{1}(\Gamma)$ est sans torsion et $X$ ou $Y$ est un $\Gamma$-espace libre, $F_{\Gamma}^{*}(X, Y)=F_{\Gamma}^{*}(X \times Y)$. Soit donc $\Gamma$ un groupe de Lie compact tel que $\pi_{1}(\Gamma)$ soit sans torsion; si $X$ et un $\Gamma$-espace libre, alors, pour tout sous-groupe $\Lambda \leq \Gamma$, il existe une suite spectrale :

$$
E_{2}^{* *}=\operatorname{Tor}_{R(\Gamma)}^{* *}\left(K_{\Gamma}^{*}(X), R(\Lambda)\right) \Longrightarrow K_{\Lambda}^{*}(X)
$$

Sur ce, on a le théorème suivant.

THÉORÈME 2.1. - Si $\pi_{1}(\Gamma)$ est sans torsion et si $X$ est un $\Gamma$-espace libre tel que $K_{\Gamma}^{*}(X)$ soit de type fini sur $R(\Gamma)$, alors $X$ vérifie la condition $(N)$. 
Preuve. - Comme la suite spectrale

$$
E_{2}^{* *}=\operatorname{Tor}_{R(\Gamma)}^{* *}\left(K_{\Gamma}^{*}(X), R(\Lambda)\right) \Longrightarrow K_{\Lambda}^{*}(X)
$$

est convergente, il suffit de voir que $\operatorname{Tor}_{R(\Gamma)}^{* *}\left(K_{\Gamma}^{*}(X), R(\Lambda)\right)$ est de type fini sur $R(\Gamma)$, car $R(\Gamma)$ est noethérien. Or $R(\Lambda)$ est de type fini sur $R(\Gamma)$ d'après [S2, $\S 3.2]$, et $K_{\Gamma}^{*}(X)$ l'est par hypothèse. On conclut grâce à [R, $\left.\S 9.21\right]$.

Proposition 2.2.- Soit $Z$ un $\Gamma$-espace compact muni d'un point de base. Si,

$$
\forall \Lambda \leq \Gamma, \quad \operatorname{ker}\left(K_{\Lambda}^{*}(Z) \longrightarrow R(\Lambda)\right)
$$

est formé d'éléments nilpotents, alors $M=M(Z)$ vérifie la condition $\left(R_{\mathcal{F}}\right)$.

Preuve. - D'après [G, sect. I, $\S 1.2 .7]$, on a une bijection :

$$
\operatorname{Spec} M_{\Lambda} \longrightarrow \operatorname{Spec} R(\Lambda), \quad \forall \Lambda \leq \Gamma .
$$

On procède alors comme dans [J, § 4.3].

Corollaire 2.3. - Soit $Z$ un $\Gamma$-espace trivial (compact) connexe. Alors $M=M(Z)$ vérifie la condition $\left(R_{\mathcal{F}}\right)$.

Preuve. - Il suffit de montrer que

$$
\forall \Lambda \leq \Gamma, \quad \operatorname{ker}\left(K_{\Lambda}^{*}(Z) \longrightarrow R(\Lambda)\right)
$$

est formé d'éléments nilpotents. Soit donc $\zeta \in K_{\Lambda}^{*}(X)$ tel que $\zeta_{+}=0$. L'ensemble $\left\{z \in Z \mid \zeta_{z}=0\right\}$ est à la fois ouvert et fermé, et est donc égal à $Z$. Finalement, $\zeta \in K_{\Lambda, 1}^{*}(Z)$ dont les éléments sont nilpotents [S1, § 5.1].

De façon générale, pour qu'un $\Gamma$-espace $Z$ tel que dans la proposition 2.1 vérifie :

$$
\forall \Lambda \leq \Gamma, \quad \operatorname{ker}\left(K_{\Lambda}^{*}(Z) \longrightarrow R(\Lambda)\right)
$$

est formé d'éléments nilpotents (et est donc nilpotent car $K_{\Lambda}^{*}(Z)$ est devenu noethérien), il suffit d'avoir la condition géométrique : pour tout $\zeta \in K_{\Lambda}^{*}(Z)$ et pour tout $z \in Z$, il existe un ouvert $U_{z}$ tel que pour tout $z^{\prime} \in U_{z}$, $\zeta_{z^{\prime}}=\zeta_{z}$; ce qui rend l'application $\zeta: Z \rightarrow \bigcup_{z \in Z} R\left(\Lambda_{z}\right)$ continue et localement constante. La connexité de $Z$ achève alors la preuve. La condition géométrique est en particulier réalisée si $Z$ est $\Gamma$-trivial, car alors $\Lambda_{z^{\prime}}=\Lambda_{z}$ sur un $U_{z}$; la proposition $[\mathrm{S} 1, \S 1.2]$ nous permet de conclure. 
Dans son article [B], A. Bojanowska a généralisé aux anneaux de la $K$ théorie équivariante la notion de support d'un idéal premier de l'anneau de représentation, qui a été inaugurée par [S2].

DÉfinition 2.4. - Soit $C$ la famille des sous-groupes cycliques de $\Gamma$. Soit $X$ un $\Gamma$-espace. On définit la catégorie $C(\Gamma, X)$ comme suit : les objets de $C(\Gamma, X)$ sont les paires $(\Sigma, c)$ où $\Sigma \in C$ et $c \subset X^{\Sigma} \neq \emptyset$ est une composante connexe.

$$
\operatorname{Mor}\left((\Sigma, c),\left(\Sigma^{\prime}, c^{\prime}\right)\right)=\left\{\gamma \in \Gamma \mid \gamma \Sigma \gamma^{-1} \subset \Sigma^{\prime}, \gamma(c) \supset c^{\prime}\right\} / \sim
$$

où $\gamma \sim \gamma^{\prime}$ si et seulement si pour tout $\sigma \in \Sigma, \gamma \sigma \gamma^{-1}=\gamma^{\prime} \sigma \gamma^{\prime-1}$. On notera

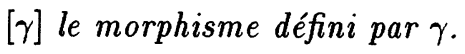

DÉfinition 2.5.- Soit $X$ un $\Gamma$-espace, et soit $(\Sigma, c)$ un objet de $C(\Gamma, X)$.

On note $i_{\Sigma, c}: \operatorname{Spec} R(\Sigma) \rightarrow \operatorname{Spec} K_{\Gamma}^{*}(X)$ l'application induite par l'inclusion de l'orbite $\Gamma / \Sigma$ dans $X$. Soit $p \in \operatorname{Spec} K_{\Gamma}^{*}(X)$. On dit que l'objet $(\Sigma, c)$ de $C(\Gamma, X)$ est un support de l'idéal $p$ si :

(a) il existe un idéal $q \in \operatorname{Spec} R(\Sigma)$ tel que $i_{\Sigma, c}(q)=p$;

(b) il n'existe pas un sous-groupe propre $\Sigma_{1} \subset \Sigma$ et un idéal $q_{1} \in$ Spec $R\left(\Sigma_{1}\right)$ tel que $i_{\Sigma_{1}, c_{1}}\left(q_{1}\right)=p$ et $c_{1}=c$.

La condition (b) étant équivalente à la suivante [B, § 4.3] :

(b') il n'existe pas de sous-groupe $\Sigma_{1} \subset \Sigma$, ni un idéal $q_{1} \in \operatorname{Spec} R\left(\Sigma_{1}\right)$ tel que $q$ soit l'image de $q_{1}$ par l'application sur les spectres induite par l'inclusion $\Sigma_{1} \subset \Sigma$.

On a alors le théorème suivant ( $\mathrm{cf} .[\mathrm{B}, \S 4.4])$.

THÉORÈme 2.6. - Soit $X$ un $\Gamma$-(ENR). Alors :

(a) chaque idéal premier de $K_{\Gamma}^{*}(X)$ a son support déterminé à un isomorphisme près dans $C(\Gamma, X)$;

(b) si $(\Sigma, c)$ est une support de l'idéal $p \in \operatorname{Spec} K_{\Gamma}^{*}(X)$ et $q, q^{\prime} \in$ $\operatorname{Spec} R(\Sigma)$ sont tels que $i_{\Sigma, c}(q)=i_{\Sigma, c}\left(q^{\prime}\right)=p$, alors il existe un morphisme $[\gamma]:(\Sigma, c) \rightarrow(\Sigma, c)$ tel que $[\gamma]_{*}(q)=q^{\prime}$. 
Soit $X$ un $\Gamma$-(ENR). Considérons, pour un sous-groupe $\Lambda$ de $\Gamma$, le morphisme de restriction

$$
K_{\Gamma}^{*}(X) \stackrel{r}{\longrightarrow} K_{\Lambda}^{*}(X)
$$

qui induit l'application

$$
r^{*}: \operatorname{Spec} K_{\Lambda}^{*}(X) \longrightarrow \operatorname{Spec} K_{\Gamma}^{*}(X) .
$$

Alors on a la proposition suivante

Proposition 2.7

(a) Soit $(P, Q) \in \operatorname{Spec} K_{\Lambda}^{*}(X) \times \operatorname{Spec} K_{\Gamma}^{*}(X)$ tel que $Q=r^{*}(P)$; soient $\left(\Sigma^{\prime}, c^{\prime}\right)$ et $\left(\Sigma^{\prime \prime}, c^{\prime \prime}\right)$ leurs supports respectifs. Alors, $\left(\Sigma^{\prime}\right)=\left(\Sigma^{\prime \prime}\right)$, où $\left(\Sigma^{\prime}\right)$ désigne la classe de conjugaison de $\Sigma^{\prime}$.

(b) Sous la condition $(N)$, si $Q \in \operatorname{Spec} K_{\Gamma}^{*}(X)$ contient le noyau

$$
I_{\Lambda}^{\Gamma}=\operatorname{ker}\left\{r: K_{\Gamma}^{*}(X) \rightarrow K_{\Lambda}^{*}(X)\right\},
$$

alors il existe un idéal $P \in \operatorname{Spec} K_{\Lambda}^{*}(X)$ tel que $Q=r^{*}(P)$.

\section{Preuve}

(a) Il existe $R^{\prime} \in \operatorname{Spec} R\left(\Sigma^{\prime}\right)$ tel que $P=i_{\Sigma^{\prime}, c^{\prime}}^{\Lambda}\left(R^{\prime}\right)$ où $i_{\Sigma^{\prime}, c^{\prime}}^{\Lambda}$ : $\operatorname{Spec} R\left(\Sigma^{\prime}\right) \rightarrow \operatorname{Spec} K_{\Lambda}(X)$. D'où $i_{\Sigma^{\prime}, c^{\prime}}^{\Gamma}\left(R^{\prime}\right)=r^{*}(P)=Q$. En outre, il existe $R^{\prime \prime} \in \operatorname{Spec} R\left(\Sigma^{\prime \prime}\right)$ tel que $i_{\Sigma^{\prime \prime}, c^{\prime \prime}}^{\Gamma}\left(R^{\prime \prime}\right)=Q$. D'après [B, $\S 4.2$ ], il existe $(\Sigma, c)$, objet de $C(\Gamma, X)$, et $R \in \operatorname{Spec} R(\Sigma)$ et aussi des morphismes

$$
\left[\gamma^{\prime}\right]:(\Sigma, c) \longrightarrow\left(\Sigma^{\prime}, c^{\prime}\right), \quad\left[\gamma^{\prime \prime}\right]:(\Sigma, c) \longrightarrow\left(\Sigma^{\prime \prime}, c^{\prime \prime}\right)
$$

tels que $\left[\gamma^{\prime}\right]_{*}(R)=R^{\prime}$ et $\left[\gamma^{\prime \prime}\right]_{*}(R)=R^{\prime \prime}$. En particulier, on a

$$
\gamma^{\prime} \Sigma \gamma^{\prime-1} \leq \Sigma^{\prime}, \quad \gamma^{\prime \prime} \Sigma \gamma^{\prime \prime-1} \leq \Sigma^{\prime \prime} .
$$

Notons $\Sigma_{1}^{\prime}=\gamma^{\prime} \Sigma^{\prime-1} \leq \Sigma^{\prime}$, alors il existe $R_{1}^{\prime} \in \operatorname{Spec} R\left(\Sigma_{1}^{\prime}\right)$ tel que $R^{\prime}$ soit l'image de $R_{1}^{\prime}$ par l'application induite sur les spectres par l'inclusion $\Sigma_{1}^{\prime} \subset \Sigma^{\prime}$. D'après la proposition 2.5 (b'), on conclut que $(\Sigma)=\left(\Sigma^{\prime}\right)$. De la même façon, on montre que $(\Sigma)=\left(\Sigma^{\prime \prime}\right)$.

(b) Comme $Q$ contient $I_{\Lambda}^{\Gamma}$, il peut être identifié à un élément de $\operatorname{Spec}\left(K_{\Gamma}^{*}(X) / I_{\Lambda}^{\Gamma}\right)$. Or $\bar{r}: K_{\Gamma}^{*}(X) / I_{\Lambda}^{\Gamma} \rightarrow K_{\Lambda}^{*}(X)$ est un monomorphisme. 
Sur la complétion de la $K$-théorie équivariante

De plus, $K_{\Lambda}^{*}(X)$ est de type fini sur $K_{\Gamma}^{*}(X) / I_{\Lambda}^{\Gamma}$ d'après la condition $(N)$. Le théorème de Cohen-Seidenberg [Bo, $\S 5.2 .1 .1]$ assure donc la surjectivité de l'application $\bar{r}^{*}$ sur les spectres.

Suivant les méthodes de $[\mathrm{J}, \S 4.3]$, on montre le théorème suivant dont la proposition 2.7 est un ingrédient important.

THÉORÈME 2.8. - Soit $X$ un $\Gamma$-(ENR). Si le foncteur $M_{\Lambda}:=K_{\Lambda}^{*}(X)$ satisfait à la condition $(N)$, alors il satisfait aussi à la condition $\left(R_{\mathcal{F}}\right)$.

\section{Références}

[A] Arouche (A.) . - $K$-théorie équivariante et théorie de complétion pour l'espace classifiant associé au lissage des actions continues d'un groupe de Lie compact, Thèse, Université de Nantes (1994).

[AM] ATIYAH (M. F.) et MACDONALD (I. G.) .- Introduction to commutative algebra, Addison-Wesley, Reading, Massachusetts, 1969.

[AS] AtiYah (M. F.) et Segal (G. B.) .- Equivariant $K$-theory and completion, J. Diff. Geom. 3 (1969), pp. 1-18.

[B] BojanowsKa (A.) . - The spectrum of equivariant $K$-theory, Math. Zeitschrift 183 (1983), pp. 1-19.

[Bo] Bourbaki (N.) .- Algèbre commutative, Chap. 5-6, Paris, Hermann, 1964.

[D] том DiEcK (T.) . - Transformation groups, Walter de Gruyter, Berlin - NewYork (1987).

[G] Grothendieck (A.) .- Eléments de géométrie algébrique, Publ. Math. IHES 4 (1960).

[H] HodgKIN (L.) .- The equivariant Künneth theorem in $K$-theory, Lecture Notes in Maths, Springer-Verlag, 496 (1976), pp. 1-100.

[J] JACKOWSKI (S.) . - Families of subgroups and completion, J. Pure Appl. Algebra 37 (1985), pp. 167-179.

[R] Rotman (J. J.) .- An introduction to homological algebra, Academic Press, New-York, 1979.

[S1] Segal (G. B.) .- Equivariant K-theory, Publ. Math. IHES 34 (1968).

[S2] SEGAL (G. B.) .- The representation ring for a compact Lie group, Publ. Math. IHES 34 (1968). 